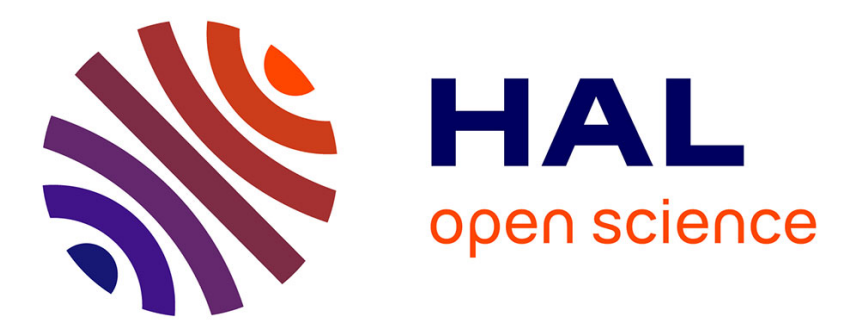

\title{
Convolution approximation for the energy loss, ionization probability and straggling of fast ions \\ P.L. Grande, G. Schiwietz
}

\section{To cite this version:}

P.L. Grande, G. Schiwietz. Convolution approximation for the energy loss, ionization probability and straggling of fast ions. The Seventh International Symposium on Swift Heavy Ions in Matter, Jun 2008, Lyon, France. hal-00207509

\section{HAL Id: hal-00207509 \\ https://hal.science/hal-00207509}

Submitted on 28 Jul 2008

HAL is a multi-disciplinary open access archive for the deposit and dissemination of scientific research documents, whether they are published or not. The documents may come from teaching and research institutions in France or abroad, or from public or private research centers.
L'archive ouverte pluridisciplinaire HAL, est destinée au dépôt et à la diffusion de documents scientifiques de niveau recherche, publiés ou non, émanant des établissements d'enseignement et de recherche français ou étrangers, des laboratoires publics ou privés. 


\title{
Convolution approximation for the energy loss, ionization probability and straggling of fast ions
}

\author{
P. L. Grande \\ Instituto de Física da Universidade Federal do Rio Grande do Sul, \\ Avenida Bento Gonçalves 9500, 91501 - 970, Porto Alegre, RS, Brazil \\ G. Schiwietz \\ Helmholtz-Zentrum Berlin f. Materialien u. Energie, \\ Abteilung SF8, Glienicker Str. 100, 14109 Berlin, Germany
}

(Dated: December 16, 2008)

\begin{abstract}
In this work we describe an extension of the convolution approximation for the ionization probability and energy-loss straggling as a function of the impact parameter for swift ions. Analytical formulas for these quantities are derived and compared to full first-order Born calculations. The physical inputs of the model are the electron density and oscillators strengths of the target as well as the screening function of the projectile (in the case of dressed ions). A very good agreement is obtained for all impact parameters. In addition, we propose a general schema to add contributions from distant and close collisions. In this way physical processes arising from large and small impact-parameters can be easily included into a single expression valid for all impact parameters. This model is then used to investigate the projectile-charge $q$ dependence of ionization, stopping and straggling cross-sections.
\end{abstract}

PACS numbers: $34.50 . \mathrm{Bw}, 34.50 . \mathrm{Fa}, 34.10 .+\mathrm{x}, 61.85 .+\mathrm{p}$

\section{INTRODUCTION}

The slowing down of fast ions in matter is dominated by electronic processes namely target ionization/excitation, projectile ionization/excitation and electron capture [1]. The basic physical quantities used to describe such electronic processes are single-event probabilities, mean energy loss and energy loss fluctuation. For gas targets or amorphous materials the related crosssections are more meaningful. However, for crystalline targets [2] as well as for the description of processes involving many electrons within the independent particle model [3] the accurate determination of the probabilities and energy loss as a function of the impact parameter is required. In particular the extension of nuclear track models to crystalline materials, to explain e.g. the formation of chains of nanodots by heavy ion irradiation as observed recently [4], is needed.

The determination of the impact-parameter-dependent ionization probability, mean energy loss and straggling is in general a very complex task, and even in the framework of the first-order Born and independent particle model ends up in a large-scale calculation. Nevertheless, for the mean energy loss only, an analytical formula has been proposed recently, which is able to reproduce full first-order and coupled-channel calculations in the so-called perturbative [5] (PCA) and unitary [6] (UCA) convolution approximation respectively (for a review in connection with heavy ions see refs [7-9]).

In this work we extend the PCA model [5] to calculate the moments of electronic energy-loss distribution as well, namely the ionization probability $\left(P_{\text {ion }}(b)\right)$ and energy-loss fluctuation $\left(\bar{Q}^{2}(b)\right)$ as a function of the impact-parameter $b$. As in ref [10], the physical input is the one underlying Bethe's theory. Thus, the validity range of the present model is limited by $Z / v<1$ ( $Z$ is the projectile-nuclear charge and $v$ is the projectile speed in atomic units). This model approaches full first-order calculations for all impact parameters without large-scale calculations. The physical inputs are the target electronic density, the oscillator strengths for each allowed optical transition and the projectile screening function in the case of projectiles carrying electrons. With the present model we have also investigated the projectile-charge $q$ dependence of the ionization, stopping and straggling cross-sections. If not indicated otherwise, atomic units $\left(e=1, m_{e}=1, \hbar=1\right)$ are used throughout the paper.

\section{MODEL}

According to the impact-parameter method [11] the ion in an ion-atom collision following a classical trajectory determined by an impact parameter $\vec{b}$ provides a time-dependent perturbation on the target electrons. Neglecting capture processes that are of minor importance at high velocities, the amplitude for a given electronic transition from the ground-state $|0\rangle$ (with energy $\varepsilon_{0}$ ) to a final target state $|f\rangle$ (with energy $\varepsilon_{f}$ ) is given in firstorder perturbation theory by [1]

$$
a_{f}(\vec{b})=-i \int_{-\infty}^{\infty} d t e^{i\left(\epsilon_{f}-\epsilon_{0}\right) t}<f|V(\vec{r}-\vec{R}(t))| 0>.
$$

Here $V(\vec{r}-\vec{R}(t))$ is the interaction potential between the projectile ion and a target electron. Only one active electron is considered and the other ones are treated in the framework of the independent particle model. Except for extraordinary small impact parameters or very low speeds, the ion trajectories are generally well described by straight lines $R(t)=\vec{v} t+\vec{b}, \vec{v}$ being the ion velocity. 
The probability for a electronic transition to a final state $f$ will be then $\left|a_{f}\right|^{2}$ and for an energy transfer $T=$ $\varepsilon_{f}-\varepsilon_{0}$ will be

$$
\frac{d P}{d T}(b)=\sum_{f}\left|a_{f}(b)\right|^{2} \delta\left(T-\left(\epsilon_{f}-\epsilon_{0}\right)\right)
$$

which involves a sum over all final target states (mostly continuum states). From this expression we can obtain the mean ionization probability $P_{\text {ion }}(b)$, mean energy loss $Q(b)$ and mean squared energy loss $Q^{2}(b)$ as follow

$$
\begin{aligned}
P_{\text {ion }}(b) & =\int_{T>I_{b}} d T \frac{d P}{d T}(b), \\
Q(b) & =\int d T T \frac{d P}{d T}(b), \\
\bar{Q}^{2}(b) & =\int d T T^{2} \frac{d P}{d T}(b),
\end{aligned}
$$

where $I_{b}$ is the binding energy $\left(I_{b}=-\epsilon_{0}\right)$. An expression similar to Eq.(3) will be also used for the total reaction probability, $P_{t o t}(b)$, but with $T>\omega_{\text {min }}$, where $\omega_{\text {min }}$ is the minimum energy transfer.

Even in a first-order treatment, the direct calculation of the above quantities demands an considerable computational effort. Therefore, we search for an approximate solution of these quantities without being a large-scale calculation. In a recent work [5] this goal has been already achieved for the mean energy loss $Q(b)$. The analytical formula derived in [5] virtually reproduces full first-order calculations for all impact parameters. However, an extension of this model for $P_{i o n}(b)$ and $\bar{Q}^{2}(b)$ is not easily made. This is due to the fact that the interpolation schema between distant and close collisions used in ref [5] is only realizable for the mean energy loss $Q(b)$.

In the following we will proceed as in ref [5] by dividing the ion-atom collisions essentially into distant and close collisions but using another interpolation schema which is more general and can be used for the energy loss distribution $d P / d T(b)$ itself.

At large impact parameters the so-called dipole approximation $[12,13]$ for $V(\vec{r}-\vec{R}(t))$ can be used, where

$$
V(\vec{r}-\vec{R}(t)) \approx V(\vec{r}-\vec{R}(t))_{\left.\right|_{\vec{r}=0}}+\vec{r} \cdot \vec{\nabla}_{\vec{r}} V(\vec{r}-\vec{R}(t))_{\left.\right|_{\vec{r}=0}}
$$

and thus, an analytical expression [13] for $d P / d T(b)$ may be obtained in the form

$$
\left(\frac{d P}{d T}(b)\right)_{\text {dipole }}=\frac{1}{2} \sum_{j} \frac{f_{j}}{T}|\hat{E}(b, T)|^{2} \delta\left(T-\omega_{j}\right)
$$

with $\hat{E}(b, \omega=T)$ being the Fourier transform of the projectile electrical field $\left(\vec{E}(b, t)=-\nabla_{\vec{r}} V(\vec{r}-\vec{R}(t))_{\left.\right|_{\vec{r}=0}}\right)$ and $\omega_{j}$ are the transition energies $\left(\omega_{j} \equiv \epsilon_{j}-\epsilon_{0}\right)$. The symbols $f_{j}$ are the well known dipole-oscillator strengths ( $\left.f_{j}=2|<j| z|0>|^{2}\left(\epsilon_{j}-\epsilon_{0}\right)\right)$, which fulfill the sum rule $\sum_{j} f_{j}=1[14]$.

For bare projectiles with nuclear charge $Z$ the interaction potential is just the Coulomb one and the square modulus of the Fourier transform of the electric field will read

$$
|\hat{E}(b, \omega)|^{2}=2 \frac{2 Z^{2}}{v^{2} b^{2}} g\left(\frac{\omega b}{v}\right)
$$

with

$$
g(x)=x^{2}\left(K_{0}^{2}(x)+K_{1}^{2}(x)\right) .
$$

$K_{0}$ and $K_{1}$ are the modified Bessel functions. Other expressions for the function $g(x)$ are presented for different projectile interaction potentials in the Appendix. As a general behavior, the function $g(\omega b / v)$ tends to the value of 1 for small impact parameters and approaches zero exponentially at large impact parameters. The solution given by Eq.(7) is exact for asymptotically large values of $b$, but it is completely inadequate for small impact parameters, where other multipole terms gain importance.

For small impact parameters the influence of the target potential can be neglected at high projectile energies [15]. Thus, the three-body problem (active electron, effective target and projectile) is reduced to a two-body one namely the scattering of the active electron described by a wavepacket corresponding to the ground-state wavefunction $\phi_{0}(\vec{r})=\langle\vec{r} \mid 0\rangle$ by the projectile potential. This problem can be solved either in the projectile reference frame [16] or in the frame where the target atom is initially at rest. In the latter case, Eq.(1) can be worked out in the following way

$$
\begin{aligned}
a_{f}(\vec{b}) & =-i \int_{-\infty}^{\infty} d t e^{i \omega_{f} t}\langle f|V(\vec{r}-\vec{R}(t))| 0\rangle \\
& =-i \int_{-\infty}^{\infty} d t \frac{e^{i \omega_{f} t}\left\langle f\left|\left[H_{0}-\epsilon_{0}, V(\vec{r}-\vec{R}(t))\right]\right| 0\right\rangle}{\omega_{f}}(1)
\end{aligned}
$$

where $H_{0}$ is the target Hamiltonian. An analytical formula for $d P / d T$ can be obtained by replacing the final target-continuum states by plane waves with momentum $\vec{k}$ in Eq.(11). This approximation is more suitable in expression in Eq.(11) than in the original expression Eq.(1), because a constant potential leads to wrong probability transitions in Eq.(1) but not in Eq.(11) since plane waves are not orthogonal to the ground-state wavefunction. By combining Eqs $(1,2)$ and (11) in order to obtain $\left|a_{f}\right|^{2}$ (or $d P / d T)$ we note that an integration over the orientation of $\vec{k}$ leads to the following integral

$$
\int d \Omega_{k} e^{i \vec{k} \cdot\left(\vec{r}_{1}-\vec{r}_{2}\right)}=4 \pi \frac{\sin \left(k\left|\vec{r}_{1}-\vec{r}_{2}\right|\right)}{k\left|\vec{r}_{1}-\vec{r}_{2}\right|},
$$

which deviates significantly from zero only for the very narrow range $\left|\vec{r}_{1}-\vec{r}_{2}\right|<1 / k$. Since at high velocities large values of $k$ dominate the integral, some unwanted commutators drop out and the energy transfer probabil- 
ity will then read

$\left(\frac{d P}{d T}(b)\right)_{\text {close }}=\frac{1}{2} \int \frac{d^{3} k}{(2 \pi)^{3}} \frac{1}{T}|<\vec{k}| \hat{E}_{\vec{r}}(b, T)|0>|^{2} \delta\left(T-\frac{k^{2}}{2}\right)$

where $\hat{E}_{\vec{r}}(b, \omega=T)$, differently from previous $\hat{E}(b, \omega)$ is the Fourier transform of the projectile electrical field at the electron position $\vec{r}$

$$
\hat{E}_{\vec{r}}(\omega)=\int_{-\infty}^{\infty} d t-\nabla_{\vec{r}} V(\vec{r}-\vec{R}(t)) e^{i \omega t}
$$

Eq.(13) can be worked out using the coordinate representation. The electronic ground-state wave function $\phi_{0}\left(\vec{r}_{2}\right)$ can be then replaced by $\phi_{0}\left(\vec{r}_{1}\right)$ because, according to Eq.(12), only $\vec{r}_{2} \approx \vec{r}_{1}$ contributes to the integral result. In fact, this peaking approximation removes the exact treatment of the momentum distribution of the bound electron. Thus, the energy transfer probability will read

$$
\left(\frac{d P}{d T}(b)\right)_{\text {close }}=\int d^{2} r_{\perp} \mathcal{P}_{\text {close }}\left(T, \vec{b}-\vec{r}_{\perp}\right) \int d z \rho\left(\vec{r}_{\perp}, z\right)
$$

with

$$
\mathcal{P}_{\text {close }}(T, b)=\frac{2 Z^{2}}{v^{2} b^{2}} \frac{C\left(2 v b, \sqrt{\frac{T}{2 v^{2}}}\right)}{\sqrt{8 v^{2}} T^{\frac{3}{2}}} .
$$

For bare projectiles the function $C$ reads

$$
\begin{aligned}
C(x, y)= & x^{2}\left(y^{3} J_{0}\left(x y \sqrt{1-y^{2}}\right) K_{0}\left(y^{2} x\right)\right. \\
& \left.+y^{2} \sqrt{1-y^{2}} J_{1}\left(x y \sqrt{1-y^{2}}\right) K_{1}\left(y^{2} x\right)\right) .
\end{aligned}
$$

Expressions of the function $C(x, y)$ for other projectile interaction potentials are presented in the Appendix. For the mean energy loss $Q(b)$ (see Eq.(4)), the corresponding expression is the same as in refs. [5, 16, 17].

The results from Eq.(7) and Eq.(13) are only valid for large and small impact parameters respectively. In what follows we propose the following interpolation ansatz,

$$
\frac{d P}{d T}(b)=\int d^{2} r_{\perp} \mathcal{P}\left(T, \vec{b}-\vec{r}_{\perp}\right) \int d z \rho\left(\vec{r}_{\perp}, z\right)
$$

with

$$
\mathcal{P}(T, b)=\mathcal{P}_{\text {close }}(T, b) F_{1}(b)+\left(\frac{d P}{d T}(T, b)\right)_{\text {dipole }} F_{2}(b)
$$

where the functions $F_{1}(b)$ and $F_{2}(b)$ are connecting functions, which must satisfy the following conditions : $F_{1}(b) \rightarrow 0$ for $b \rightarrow \infty$ and $F_{2}(b) \rightarrow 0$ for $b \rightarrow 0$. Thus, the close and distant collisions contributions are turned on/off as a function of the impact parameter. The first term in Eq. (19) describes violent binary collisions and the last term accounts for the long ranged dipole transitions. For latter case we note that for large impact parameters ( $\mathrm{b}>>\mathrm{r}_{\text {shell }}, \mathrm{r}_{\text {shell }}$ being the shell radius) the convolution from Eq.(18) disappears since the range of perpendicular distances $r_{\perp}$, where the electron density is significant, is small compared to $\mathrm{b}$ and the function $\mathcal{P}\left(\vec{b}-\vec{r}_{\perp}\right)$ is not sensitive to small variation of $r_{\perp}$ at high velocities. Hence, Eq. (18) agrees with the results obtained from the dipole approximation given by Eq.(7).

The expressions for the ionization probability, energy loss and straggling as a function of the impact parameter using the definitions from Eqs.(3-5) and the the present model for $d P / d T$ (Eq.(18)) will then read

$$
\begin{gathered}
P_{\text {ion }}(b)=\int d^{2} r_{\perp} \mathcal{K}^{(0)}\left(\vec{b}-\vec{r}_{\perp}\right) \int d z \rho\left(\vec{r}_{\perp}, z\right) \\
Q(b)=\int d^{2} r_{\perp} \mathcal{K}^{(1)}\left(\vec{b}-\vec{r}_{\perp}\right) \int d z \rho\left(\vec{r}_{\perp}, z\right) \\
\bar{Q}^{2}(b)=\int d^{2} r_{\perp} \mathcal{K}^{(2)}\left(\vec{b}-\vec{r}_{\perp}\right) \int d z \rho\left(\vec{r}_{\perp}, z\right)
\end{gathered}
$$

with

$$
\begin{aligned}
\mathcal{K}^{(n)}(b) & =\frac{2 Z^{2}}{v^{2} b^{2}}\left(\left(2 v^{2}\right)^{n-1} h^{(n)}(2 v b) F_{1}(b)\right. \\
& \left.+\sum_{j} f_{j} \omega_{j}^{n-1} g\left(\frac{\omega j b}{v}\right) F_{2}(b)\right),
\end{aligned}
$$

and

$$
h^{(n)}(2 v b)=\left(2 v^{2}\right)^{1-n} \int d T T^{n} \frac{C\left(2 v b, \sqrt{\frac{T}{2 v^{2}}}\right)}{\sqrt{8 v^{2}} T^{\frac{3}{2}}}
$$

The connecting functions $F_{1}(b)$ and $F_{2}(b)$ can be determined from the mean energy-loss formula. Recalling the interpolation procedure from ref.[5], where close and distant collisions were connected successfully through a product ansatz (using the same notation)

$$
\mathcal{K}^{(1)}(b)=\frac{2 Z^{2}}{v^{2} b^{2}}\left(h^{(1)}(2 v b) \times \sum_{j} f_{j} g\left(\frac{\omega_{j} b}{v}\right)\right),
$$

we have the following condition for $F_{1}(b)$ and $F_{2}(b)$

$$
h^{(1)}(2 v b) F_{1}(b)+g\left(\frac{\omega b}{v}\right) F_{2}(b)=h^{(1)}(2 v b) \times g\left(\frac{\omega b}{v}\right) .
$$

The general solution is $F_{1}(b)=g\left(\frac{\omega b}{v}\right) \times \Gamma(b)$ and $F_{2}=$ $h^{(1)}(2 v b) \times(1-\Gamma(b))$. Since for intermediate impact parameters both function $h^{(1)}$ and $g$ approach a value of 1 (ref [5]), the exact form of the function $\Gamma(b)$ is not so important (for the mean energy loss). Thus, $\Gamma(b)$ is assumed here to be of the form $\exp \left(-\alpha\left(b / b_{c}\right)^{2}\right)$, with $b_{c}$ being an intermediate impact parameter determined from the geometric mean of $1 / v$ (the de Broglie wavelength of target electron in the projectile frame) and $v / \omega_{\min }$ (the adiabatic radius). The value of the constant $\alpha$ was determined from a best fit to first-order Born results for the total reaction probability and was fixed at $\alpha=2 / 3$. It is pointed out that the connecting functions may also 


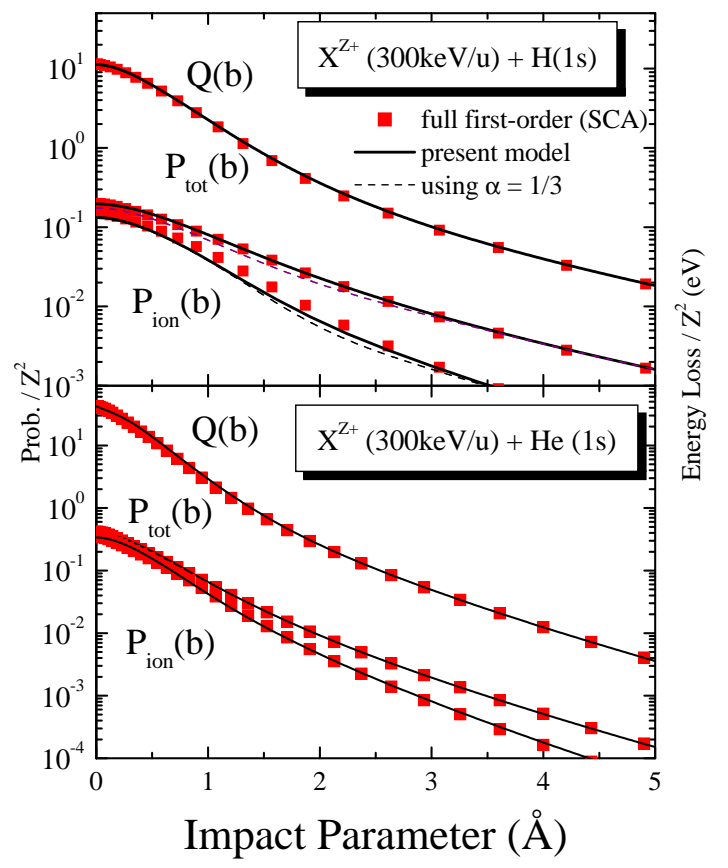

FIG. 1: The electronic energy loss $Q(b)$ (in $\mathrm{eV}$ ), total reaction probability $P_{t o t}(b)$ and ionization probability $P_{i o n}(b)$ are displayed as a function of the impact parameter for bare ions (with nuclear charge $\mathrm{Z}$ ) colliding with $\mathrm{H}$ and $\mathrm{He}$ atoms at 300 $\mathrm{keV} / \mathrm{u}$. The symbols (squares) represent full first-order calculations from ref[21] and the solid and dashed lines correspond to the present model. Note that the probabilities may exceed $100 \%$ for large nuclear charges $\mathrm{Z}$.

depend on the energy transfer $T$, but this was not considered here.

It can be shown that the present analytical formulas are consistent with the Bethe stopping [18] and Bohr straggling[19] formulas at high velocities respectively. Therefore, for asymptotically high projectile energies the present model gives the exact limiting impact-parameter dependencies of the electronic energy loss and energy straggling. Moreover, the exact definition of the borderline between close and distant collision is of little importance as demonstrated by Fano [20]. Thus, the exact form of the connecting functions is not crucial. It is also pointed out that the present formulas as given above are strictly valid only for a one-electron system. In the framework of the independent-particle model, however, we can still use the results of Eq.(18), but we have to consider the electronic density and the oscillators strengths for each electron of all occupied target shells.

\section{COMPARISON WITH FULL FIRST-ORDER CALCULATIONS}

In figures 1 to 3 we show the results of the present model for the total reaction and ionization probabilities and energy-loss fluctuation of ions colliding with $\mathrm{H}, \mathrm{He}$ and $\mathrm{C}$ targets (solid lines) in comparison with full first-

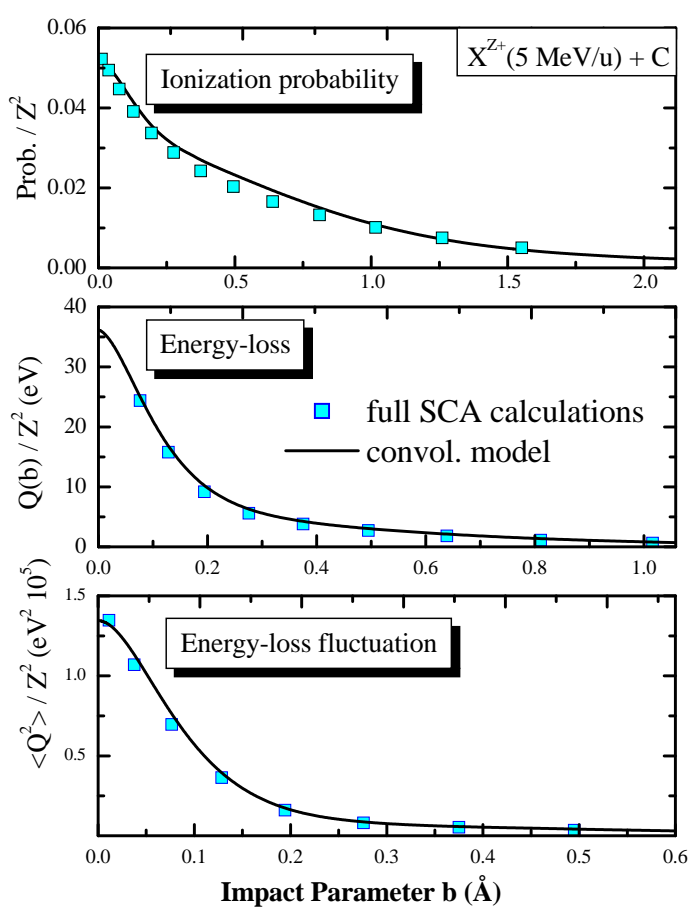

FIG. 2: The electronic energy loss $Q(b)$ (in eV), ionization yield and mean squared energy loss are displayed as a function of the impact parameter for $5 \mathrm{MeV} / \mathrm{u}$ bare ions colliding with $\mathrm{C}$ atoms. The symbols represent full first-order calculations from ref[21] and the solid lines correspond to the present model.

order calculations[21] (symbols). First-order perturbation theory yields reliable results at $Z / v<1$, where the condition of a small perturbation is fulfilled. All input parameters (electronic densities and oscillator strengths) were obtained from analytical formulas for the $\mathrm{H}$ target and from Hartree-Fock-Slater wavefunctions for He and $\mathrm{C}$ $[21,22]$. It is pointed out that the present full first-order Born results agree with the results from the pioneering works of Kabachnick and coworkers [10].

Fig. 1 shows a comparison for the impact parameter dependence of incident bare ions at $300 \mathrm{keV} / \mathrm{u}$ with two different targets $(\mathrm{H}$ and $\mathrm{He})$. In all cases we obtain a very good agreement with full SCA calculations. The same agreement is observed for other higher projectile velocities. The largest deviation of only about $10 \%$ is found for the ionization probability of $\mathrm{H}$ at impact parameters around $1.5 \AA$. The ionization probability has a pronounced maximum at small impact parameters. However, this does not mean that these small impact parameters will dominate the ionization cross-section. In fact, for the He target example, impact parameters larger than the He shell radius contribute mostly to the ionization cross-section. Fig. 1 also shows the present calculations with another constant $\alpha$ (a factor of two smaller) for the interpolation function $\Gamma(b)$. It can be seen that the results are in general very insensitive to the choice of the weighting function $\Gamma(b)$.

The results of the present model are shown in Fig. 2 for 
bare ions at $5 \mathrm{MeV} / \mathrm{u}$ colliding with a $\mathrm{C}$ atom, which has three sub-shells. Calculations for the ionization probability $\left(P_{\text {ion }}(b)\right)$, mean energy-loss $(Q(b))$ energy-loss fluctuation $\left(Q^{2}(b)\right)$ have been performed for each $\mathrm{C}$ sub-shell. The agreement of present model (solid lines) with full SCA calculations (squares) is very good, in particular for $Q(b)$ and $Q^{2}(b)$. It is pointed out that the present model as well as the full SCA calculations account for the Pauli exclusion principle. This means that transitions to occupied sub-shells are explicitly excluded. The Pauli principle is taken into account by our present model by using an appropriate set of oscillator strengths and energy transfers in Eq.(18).

The energy dependence of the electronic straggling $\left(W=\int 2 \pi b d b \bar{Q}^{2}(b)\right)$ is presenteds in Fig. 3 for bare ions colliding with atomic hydrogen. The results are compared with plane-wave Born approximation (PWBA) $[14,23]$ and with the Bohr straggling [19]. Although the PCA and PWBA rely on the Born approximation they may differ because of the additional approximations performed to derive the PCA model. Here we have also used the kinematical correction from ref [24] in order to extend the present PCA model down to lower energies. This correction is the main contribution to the so-called shellcorrection in stopping theory [24] and has been adopted by replacing the close-collision contribution $h^{(n)}$ by the value $\left\langle h^{(n)}>\right.$ averaged over the momentum distribution of the target electron (the Comptom profile) according to formulas from ref. [24]. Thus, the information on the target electron velocity, which was destroyed by the peaking approximation used to derive the convolution approximation is partially restored. It is pointed out that the way to average the close-collision contribution is different for probability (or cross-section), energy-loss (stopping cross-section) and mean energy-loss squared (energy straggling cross-section). As can be observed from this

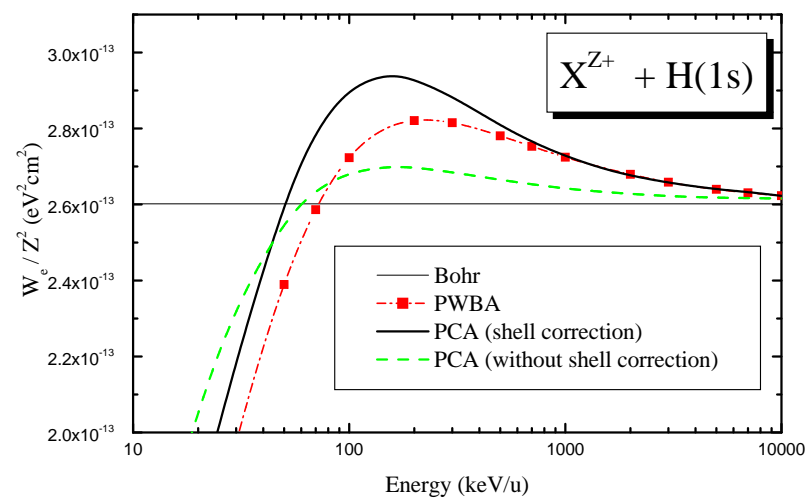

FIG. 3: The straggling cross-section for bare ions in $\mathrm{H}(1 \mathrm{~s})$ as a function of the projectile energy. The symbols correspond to PWBA [23] calculations from ref[21] and the solid lines correspond to the present model with and without kinematical correction from [24]. Note the strong zero-suppression of the straggling values. figure, the inclusion of the kinematical corrections for the electronic straggling turns out to be very important for an accurate description of the overshooting (over the Bohr straggling value) part of the energy-loss straggling [25]. In fact, since the energy-loss straggling is much more sensitive to close than to distant collisions, this correction is more important than the one corresponding to the case of stopping or ionization cross-sections. Conversely, the kinematical correction is not so important for the ionization cross-section since it is dominated by distant collisions.

\section{PROJECTILE CHARGE DEPENDENCE}

In order to obtain the projectile-charge $q$ dependent ionization, stopping and straggling cross-sections, we have assumed the following screened projectile potential $V_{p}$

$$
V_{p}(\vec{r})=-\left(\frac{Z-n_{e}}{r}+\frac{n_{e}}{r} \Phi(r)\right)
$$

where $n_{e}$ is the number of electrons carried by the projectile $\left(n_{e}=Z-q\right)$, and $\Phi(r)$ is the projectile screening function, which is obtained from

$$
\Phi(r)=\Phi_{\text {neutral }}(r / a),
$$

with an approximate screening length $a=1-q / Z$ or $\left(a=n_{e} / Z\right)$ (taken from ref.[27]), where $\Phi_{\text {neutral }}$ is the screening function for the corresponding neutral projectile. It has been tabulated for all elements, for instance using Dirac-Hartree-Fock-Slater (DHFS) calculations, in ref.[28], as a sum of exponentials functions. The analytical formulas for the distant and close collision contributions for a screened projectile are given in the Appendix.

Fig. 4 shows the projectile-charge dependence of the ionization, stopping and straggling cross-sections for $\mathrm{Ne}$ ions at $60 \mathrm{MeV} / \mathrm{u}$ colliding with $\mathrm{Xe}$ atoms $(Z / v \approx 0.2)$. Also shown are curves proportional to $q^{2}$. As can be observed from this figure, the $q^{2}$ scaling, largely used for the stopping power calculations, fails to describe the present stopping and straggling calculations. Only the $q$ dependent ionization cross-section can be reasonable well approximated by a $q^{2}$ law (for a discussion on and use of the $q^{2}$ dependence see ref. [27, 29-33]). This behavior can be explained by the fact that the ionization cross-section is dominated by distant collisions where the projectile behaves as a point charge $q$. On the other hand, the energy-loss straggling depends only on close collisions. Since the screening due to the bound projectile electrons is not totally effective for close collisions, the energy-loss straggling will depend much more on the projectile nuclear charge $Z$ and much less on $q$ (as can be observed from the lowest panel of Fig. 4). In the case of the stopping power distant and close collisions contribute equally at high energies. This fact is known as the Bethe equipartition rule and it leads to a reduction factor of about two in the $q$ dependence of $S_{e}$, specifically at low $q$. 


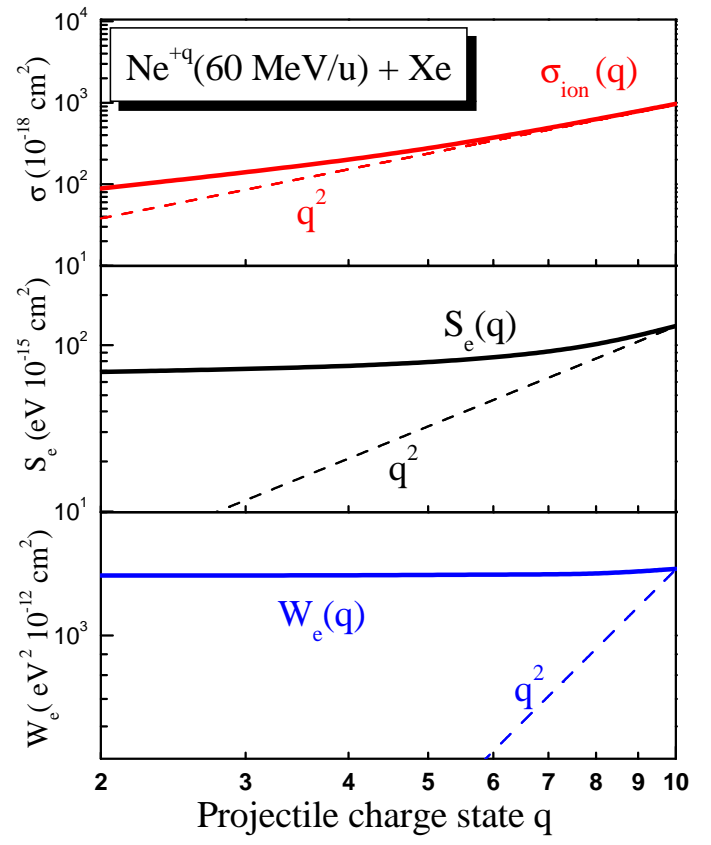

FIG. 4: Ionization, stopping and straggling cross-sections for $60 \mathrm{MeV} / \mathrm{u}$ Ne ions in Xe as a function of the projectile chargestate $q$. The solid lines correspond to the present model and the dashed-lines represent a $q^{2}$ dependence.

The $q$ dependent stopping cross-section for $2 \mathrm{MeV} / \mathrm{u} \mathrm{U}$ ions in $\mathrm{Xe}(Z / v \approx 10)$ is depicted in Fig. 5. In this case, a $q^{2}$ dependence is clearly observed for $\mathrm{U}$ ions carrying few electrons (q close to Z). In fact, the projectile electrons screen so strongly the projectile nuclear charge, that only $q$ elementary charges are left for the interaction with the Xe target electrons. Nevertheless, this case (strong screening) is only possible for $Z / v>1$, which precludes the use of the present model, since it is based on the first-order Born approximation. In fact, non-pertubative calculations must be used, as the unitary convolution approximation (UCA) $[6,34]$ (see dashed line). Because of the influence of higher-order effects, the q-dependence departs at all q significantly from the $q^{2}$ curve. In Fig. 5 we find a strong non-perturbative suppression of $S_{e}$ (exceeding a factor of 3 ) due to the non-unitary behavior of perturbation theory (ionization yields in perturbation theory may exceed 1 per initial target electron). This non-unitary does not show up in the UCA results and of course it is also absent in exact quantum mechanical treatments such as coupled-channel calculations [6]. At higher speeds or lower $\mathrm{Z}$ values we expect an additional flattening of the $q$ dependence of $S_{e}$, due to the Bloch terms and due to the projectile ionization contribution. Generally, a $q^{2}$ scaling (as it is often used in literature [33]) can only approximately be valid for very restricted parameter regions as for example in the experiments involving planar channeling by Golovchenko et al. [29] where a complete cancellation of higher-effects has been reported $[29,35,36]$.

\section{CONCLUSIONS}

Analytical formulas for the ionization/total reaction probabilities, electronic energy loss and energy straggling as a function of the impact parameter are developed that are valid at high energies and for a wide range of impact parameters. For the mean energy loss and mean squared energy loss, the integral over all impact parameters recovers the Bethe formula and Bohr straggling formula respectively.

The physical input of the model are the projectile screening function, in the case of dressed projectiles, electron density and the set oscillator strengths for each sub-shell. A general interpolation procedure between close and distant collisions has been proposed by introducing additive connecting functions. Thus, expressions derived for small and large impact parameters can be smoothly joined with good precision at high projectile energies. Comparisons with full first-order calculations (SCA) show that the present model yields reliable values of probabilities, energy loss, and straggling as a function of the impact parameters.

We have also discussed the projectile charge-state dependence of ionization, stopping and straggling crosssections. In particular for the stopping cross-section, the use of the $q^{2}$ scaling has to be avoided. The contribution of projectile bound-electrons is responsible for the deviation of the $q^{2}$ dependence. Only in the case of strongly screened projectiles, the $q^{2}$ scaling of the stopping power could be expected in a first-order approach. However, this condition implies that the shell radius for the projectile bound electrons $(\approx 1 / Z)$ should be much smaller than the typical minimal impact parameter $(\approx 1 / v)$ (down where the $h$ vanishes). In other words, in order to have a strongly screened projectile $Z / v$ should be larger than one, which contradicts the first-order assump-

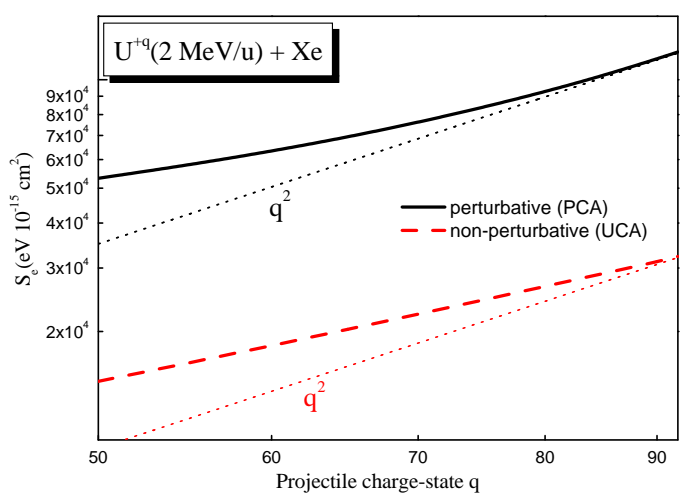

FIG. 5: The stopping cross-sections for $2 \mathrm{MeV} / \mathrm{u}$ U ions in $\mathrm{Xe}$ as a function of the projectile charge-state $q$. The solid line corresponds to the perturbative PCA model and the red dashed line to the non-perturbative UCA model. The dottedlines represent a $q^{2}$ dependence. 
tion. The same analysis can be done for non-perturbative collisions as well, where the $q^{2}$ dependence could emerge from a compensation between higher-order effects and the contribution of the nuclear charge $Z$ in the case of partial screening due to the bound electrons. Projectile screening, projectile ionization and Bloch terms lead to a reduction, specifically to a flattening of the charge-state dependence of the energy loss, as shown in this work. A possible steepening due to Barkas terms and electron capture is usually important only at lower velocities. Thus, the $q^{2}$ scaling can generally not be realized at high speeds and typical powers $x$ for $q^{x}$ are significantly below two for $S_{e}$. These powers are only slightly below two for the ionization cross-section, but close to zero for the energy-loss straggling.

\section{ACKNOWLEDGMENTS}

This work was partially supported by the Brazilian agencies $\mathrm{CNPq}$ and CAPES.

\section{APPENDIX}

Here we have considered the following projectile screening potential with

$$
\begin{aligned}
f_{1}(x)= & n_{p} / Z \sum_{i}\left(a_{i} K_{1}\left(\sqrt{x^{2}+\lambda_{i}^{2}}\right) \sqrt{x^{2}+\lambda_{i}^{2}}\right. \\
& \left.+\eta_{i} \lambda_{i} K_{0}\left(\sqrt{x^{2}+\lambda_{i}^{2}}\right)\right) \\
& +\left(1-n_{p} / Z\right) x K_{1}(x) \\
f_{2}(x) & =n_{p} / Z \sum_{i}\left(a_{i} x K_{0}\left(\sqrt{x^{2}+\lambda_{i}^{2}}\right)\right. \\
& \left.+x \eta_{i} \lambda_{i} K_{1}\left(\sqrt{x^{2}+\lambda_{i}^{2}}\right) / \sqrt{x^{2}+\lambda_{i}^{2}}\right) \\
& +\left(1-n_{p} / Z\right) x K_{0}(x) .
\end{aligned}
$$

with $\eta_{i} \equiv b_{i} b$ and $\lambda_{i} \equiv=c_{i} b$.

The function $C(x, y)$ for close collisions in Eq.(16) and for the general screening potential above reads

$$
C(x, y)=x^{2} F(y) \frac{G(x, y)}{Z^{2}}
$$

with the auxilary functions defined by:

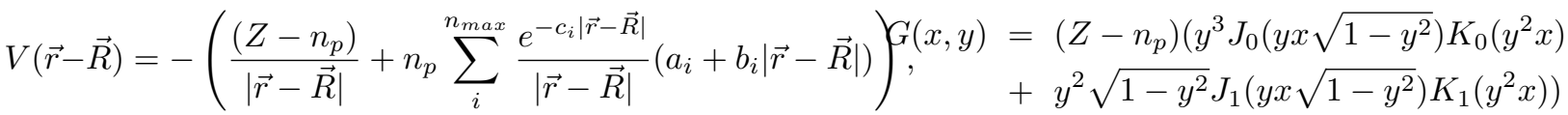

$$
\begin{aligned}
& +n_{p} \sum_{i} a_{i}\left(y^{3} J_{0}\left(y x \sqrt{1-y^{2}}\right) K_{0}\left(x \sqrt{y^{4}+\gamma_{i}^{2}}\right)\right. \\
& \left.+\sqrt{1-y^{2}} \sqrt{y^{4}+\gamma_{i}^{2}} J_{1}\left(y x \sqrt{1-y^{2}}\right) K_{1}\left(x \sqrt{y^{4}+\gamma_{i}^{2}}\right)\right) \\
& +n_{p} \sum_{i} \beta_{i} \gamma_{i} x\left(\frac { y ^ { 3 } } { \sqrt { y ^ { 4 } + \gamma _ { i } ^ { 2 } } } J _ { 0 } ( y x \sqrt { 1 - y ^ { 2 } } ) K _ { 1 } \left(x \sqrt{y^{4}+(3}\right.\right. \\
& \left.+\sqrt{1-y^{2}} J_{1}\left(y x \sqrt{1-y^{2}}\right) K_{0}\left(x \sqrt{y^{4}+\gamma_{i}^{2}}\right)\right)
\end{aligned}
$$
lations [28]. Usually, the number of single-zeta potential terms, $n_{\max }$, corresponds to the number of atomic shells of the projectile.

The corresponding expression for the function $g(x)$ (similar to the expressions for distant collisions from ref [27]) in the Eq.(8) is

$$
g(x)=f_{1}(x)^{2}+f_{2}(x)^{2}
$$

and

$F(y)=\left(Z-n_{p}\right)+n_{p} \sum_{i}\left(a_{i} \frac{y^{2}}{y^{2}+\gamma_{i}^{2}}+\beta_{i} \gamma_{i} \frac{y^{2}}{\left(y^{2}+\gamma_{i}^{2}\right)^{2}}\right)$

with $\beta_{i} \equiv b_{i} /(2 v)$ and $\gamma_{i} \equiv c_{i} /(2 v)$.

[1] M. R. C. McDowell and J. P. Coleman, Introduction to the Theory of Ion-Atom Collisions (Amsterdam: Noth Holland, 1970).

[2] D. S. Gemmell, Rev. Mod. Phys. 46, 129 (1974).

[3] N.M. Kabachnik, V.N. Kondartyev, Z. Roller-Lutz and H.O. Lutz, Phys. Rev. A 56,2848 (1997). Z. Kaliman, N. Orlic, N.M. Kabachnik and H.O. Lutz, Phys. Rev. A 65,012708 (2001).
[4] E. Akcoeltekin, T. Peters, R. Meyer, A. Duvenbeck, M. Klusmann, I. Monnet, H. Lebius and M. Schleberger, Nature Nanotechnology 2, 290 (2007).

[5] P. L. Grande and G. Schiwietz, Phys. Rev. A 58, 3796 (1998).

[6] G. Schiwietz and P. L. Grande, Nucl. Instr. and Meth. B 153, 1 (1999).

[7] P.L. Grande and G. Schiwietz, Nucl. Instr. and Meth. B 
195, 55 (2002).

[8] G. Schiwietz, M. Roth, K. Czerski, F. Staufenbiel, and P.L. Grande, Nucl. Instr. Meth. B225, 4-26 (2004) and Nucl. Instr. Meth. B226 (2004) 683-704

[9] P.L. Grande and G. Schiwietz, "Ionization and energy loss beyond perturbation theory", in "Advances in Quantum Chemistry", vol. 45, pp.7-46 (book article ed. by J. Sabin, 2004, Elsevier Inc.)

[10] N. M. Kabachnik, V. N. Kondratev, and O. V. Chumanova, Phys. Status Solidi B 145, 103 (1988); N. M. Kabachnik, V. Ya. Chumanov, O. V. Chumanova, Nucl. Instr. and Meth. 111, 22 (1996).

[11] N.F. Mott, Proc. Cambr. Phil. Soc., 27, 553 (1931); J.Bang and J.M.Hansteen, Kgl. Dan. Vidensk. Selsk. Mat. Fys. Medd. 31, No.13 (1959); L. Wilets and S. J. Wallace, Phys.Rev. 169, 84 (1968); M.R. Flannery and K.J.MacCann, Phys. Rev. A8 , 2915 (1973).

[12] J.D Jackson," Classical Electrodynamics", Chapter 13 (John Wiley and Sons, Inc. 1975).

[13] C.O. Reinhold and J.Burgdoerfer, J. Phys. B: At. Mol Opt. Phys 26, 3101 (1993).

[14] H. A. Bethe, R.W. Jackiw, "Intermediate Quantum Mechanics" (2nd edition, W.A. Benjamin, inc, 1968).

[15] F. Bloch, Ann. Physik 16, 285 (1933).

[16] V.A. Knodyrev J. Phys. B:Ar. Mol. Opt. Phys. 33, 5045 (2000).

[17] In ref. [5] was omitted a part integration in Eq.(13)

[18] H. Bethe, Ann. Physik 5, 325 (1930).

[19] N. Bohr, Philos. Mag. 25,10 (1913); N. Bohr, Phys. Rev. 59, 270 (1941).

[20] U. Fano, Penetration of protons, alpha particles, and mesons. Ann. Rev. Nucl. Sci. 13 1, (1963).

[21] The numerical procedures are same as used in G. Schiwietz and P.L. Grande, Nucl. Instr. and Meth. B69,10 (1992); P.L.Grande and G. Schiwietz, Phys. Rev.
A47,1119 (1993);P.L. Grande and G. Schiwietz, Nucl. Instr. and Meth.B132, 264 (1997).

[22] F. Herman and S. Skillmann, in Atomic Structure Calculations, (Prentice-Hall, Inc. Englewood Cliffs, New Jersey,1963).

[23] D.R. Bates and G.W. Griffing. Proc. Phys. Soc. A 68 (1955).

[24] P. Sigmund, Phys. Rev. A 26, 2497(1982).

[25] P. Sigmund, L.G. Glazov, Europ. Phys. J. D. 23, 211 (2003).

[26] P.L. Grande and G. Schiwietz, Phys. Rev. A44, 2984 (1991).

[27] P. Sigmund, Phys. Rev. A 56, 3781 (1997).

[28] F. Salvat, J.D. Martinez, R. Mayol and J. Parellada, Phys. Rev. A 36, 467 (1987).

[29] J. A. Golovchenko, A. N. Goland, J. S. Rosner, C. E. Thorn, H. E. Wegner, H. Knudsen, and C. D. Moak, Phys. Rev. B 23, 957 (1981).

[30] G. Maynard, M. Chabot and D. Gardes, Nucl. Instr. and Meth. B164-165, 139 (2000)

[31] A.F. Lifschitz and N.R. Arista, Phys. Rev. A 69, 012902 (2004).

[32] G. Zwicknagel, P.G. Reinhard, C. Seele,, C. Toepffer, Fusion Engineering and Design, vol.32-33, 523-8, (1996).

[33] W. Brandt and M. Kitagawa, Phys. Rev. B. 26, 3968 (1982).

[34] The computational implementation of the UCA method $[5,6]$, CasP (Convolution Approximation for Swift Particles) program, is available at http://www.hmi.de/people/schiwietz/casp.html.

[35] G. de M. Azevedo, P.L. Grande, M. Behar and J.F. Dias, Physical Review Letters 86, 1482 (2001).

[36] P. Sigmund and A. Schinner, Physical Review Letters 86, 1486 (2001). 